\title{
Process Capability Plots for One-Sided Specification Limits
}

\author{
Kerstin Vännman and Malin Albing \\ Department of Mathematics, Luleå University of Technology, Sweden \\ (Received December 2005, accepted May 2007)
}

\begin{abstract}
We extend the idea of process capability plots from the case of two-sided specification intervals to derive a graphical method useful when doing capability analysis having one-sided specification limits. The derived process capability plots are based on existing capability indices for one-sided specification limits. Both the cases with and without a target value are investigated. Under the assumption of normality we suggest estimated process capability plots to be used to assess process capability at a given significance level. Theoretical results are given for determining the significance level as well as power for the method. The presented graphical approach is helpful to determine if it is the variability, the deviation from target, or both that need to be reduced to improve the capability. Examples are presented.
\end{abstract}

Keywords: Capability index, graphical method, hypothesis testing, one-sided specification interval, power, process capability plot.

\section{Introduction}

process capability index is frequently used when measuring the capability of a manufacturing process. Such an index is designed to quantify the relation between the actual performance of the process and its specified requirements. For thorough discussions of different capability indices and their statistical properties see, e.g. the books by Kotz and Johnson [8] and Kotz and Lovelace [10] and the review paper with discussion by Kotz and Johnson [9].

Most of the published articles focus on the case when the specification interval is two-sided. In such a case the two most widely used capability indices in industry today are

$$
C_{p}=\frac{U S L-L S L}{6 \sigma} \text { and } C_{p k}=\frac{\min (U S L-\mu, \mu-L S L)}{3 \sigma},
$$

where $[L S L, U S L]$ is the specification interval, $\mu$ is the process mean and $\sigma$ is the process standard deviation of the in-control process. According to today's modern quality improvement theories, it is important to use target values and to keep the process on target. The indices in (1) do not take into account that the process mean, $\mu$, may differ from the target value, $T$. A capability index which does and hence can be used as a measure of process centering, is $C_{p m}$, where

$$
C_{p m}=\frac{U S L-L S L}{6 \sqrt{\sigma^{2}+(\mu-T)^{2}}} .
$$

One-sided specification intervals are also used in industry, see, e.g. Kane [7] and 
Gunter [5]. However, there have been relatively few articles in the statistical literature dealing with this case. When Kane [7] introduced $C_{p k}$ in (1) he defined that index using the following indices for one-sided specification limits:

$$
C_{P U}=\frac{U S L-\mu}{3 \sigma} \text { and } C_{P L}=\frac{\mu-L S L}{3 \sigma},
$$

for an upper and lower specification limit, USL and $L S L$, respectively. Kane [7] also introduced the following indices for one-sided specification limits with a target value $T$ :

$$
C_{P U}=\frac{U S L-T-|T-\mu|}{3 \sigma} \text { and } C_{P L}=\frac{T-L S L-|T-\mu|}{3 \sigma} .
$$

Chan et al. [2] have suggested the following generalization of $C_{p m}$ to the case where one-sided specification limits are required:

$$
C_{p m u}^{*}=\frac{U S L-T}{3 \sqrt{\sigma^{2}+(\mu-T)^{2}}} \text { and } C_{p m l}^{*}=\frac{T-L S L}{3 \sqrt{\sigma^{2}+(\mu-T)^{2}}} .
$$

In order to gain sensitivity with regard to departures of the process mean from the target value, and at the same time generalize the indices (3) - (5), Vännman [14] defined two different families of capability indices for one-sided specification intervals, depending on two parameters, $u$ and $v$, as

$$
C_{p a u}(u, v)=\frac{U S L-\mu-u|\mu-T|}{3 \sqrt{\sigma^{2}+v(\mu-T)^{2}}} \text { and } C_{p a l}(u, v)=\frac{\mu-L S L-u|\mu-T|}{3 \sqrt{\sigma^{2}+v(\mu-T)^{2}}}
$$

where $u \geq 0, v \geq 0$, and

$$
C_{p v u}(u, v)=\frac{U S L-T-u|\mu-T|}{3 \sqrt{\sigma^{2}+v(\mu-T)^{2}}} \text { and } C_{p v l}(u, v)=\frac{T-L S L-u|\mu-T|}{3 \sqrt{\sigma^{2}+v(\mu-T)^{2}}}
$$

where $u \geq 0$ and $v \geq 0$, but $(u, v) \neq(0,0)$.

The class of indices in (6) generalizes the indices in (3). They are obtained by setting $u=0$ and $v=0$ in (6). By setting $u=1, v=0$ in (7) we get the indices in (4) and with $u=0$, $v=1$ in (7) we get the indices in (5). Note that, when the process is on target, i.e. $\mu=T$, the indices in (6) and (7) simplifies to the indices in (3).

Vännman [14] also considered estimators of the proposed classes and provided, under the assumption of normality, explicit forms of the distributions of the families of the estimated indices. Furthermore, tests were derived under the assumption that the process is on target and their properties where explored for different values of the parameters. It was found that to achieve large power, when the process is on target, and at the same time have high sensitivity against departures from the process mean the index with $u=0, v=4$ in (6) and with $u=1, v=4$ in (7), respectively, is recommended.

More recently Pearn and Chen [12], Lin and Pearn [11] and Pearn and Shu [13] have studied tests and confidence intervals for the indices $C_{P U}$ and $C_{P L}$ in (3) and presented extensive tables for practitioners to use when applying these methods. 
In the case of two-sided specification limits Deleryd and Vännman [4] and Vännman [15], [16] discuss plots, based on process capability indices, which can be used to assess process capability at a given significance level. An advantage with using such process capability plots, compared to using the capability index alone, is that they will instantly give visual information, simultaneously about the location and spread of the studied characteristics, as well as information about the capability of the process. When the process is non-capable, these plots are helpful when trying to understand if it is the variability, the deviation from target, or both that need to be reduced to improve the capability.

In the present paper we study process capability plots to be used for one-sided specification limits. We present plots based on the classes of indices given in (6) and (7). Under the assumption of normality we suggest estimated process capability plots to be used to assess process capability at a given significance level. These results are more general than the tests derived by Vännman [14].

We consider, in this paper, the case with an upper specification limit, USL, only. The case with a lower specification limit is analogous. Furthermore, we will assume that the process mean $\mu$ always will be less than the upper specification limit USL.

Vännman [14] found that the statistical properties of the estimators of the indices in (6) and (7) were unsatisfactory for values of $u>1$. Furthermore, it was found sufficient to consider integer values of $u$ and $v$, since not much is gained in statistical efficiency or sensitivity against departures from the process mean when using non-integer values. Hence in this paper we will only consider indices with $u=0$ or 1 .

\section{Process Capability Plots}

For simplicity we will use the notation $C_{p u}(u, v)$ to denote either of the two classes of indices $C_{p a u}(u, v)$ in (6) and $C_{p v u}(u, v)$ in (7), unless we study a specific class of indices. When using process capability indices a process is defined to be capable if the process capability index exceeds a certain threshold value $k$. Some commonly used values are $k=1$, $k=4 / 3, k=3 / 2$, or $k=2$, see, e.g. Pearn and Chen [12]. A process is thus defined to be capable if $C_{p u}(u, v)>k$ for given values of $u, v$, and $k$.

Analogous to the case with two-sided specification limits, process capability plots can be used in order to facilitate the understanding of the restrictions that the index imposes on the process parameters $(\mu, \sigma)$. These plots are based on contour curves of the indices. For one-sided specification indices we can then express $C_{p u}(u, v)=k$ as functions of $\mu$ and $\sigma$, or as simple transformations of $\mu$ and $\sigma, \mu_{t}$ and $\sigma_{t}$, respectively, where

$$
\mu_{t}=\frac{\mu-T}{U S L-T} \text { and } \sigma_{t}=\frac{\sigma}{U S L-T} .
$$

The reason for making the contour plot as a function of $\left(\mu_{t}, \sigma_{t}\right)$ instead of a function of $(\mu, \sigma)$ is to obtain a plot where the scale is invariable, irrespective of the value of the specification limit. If the process is on target, i.e. $\mu=T$, we have $\mu_{t}=0$ and if $\mu=U S L$ we have $\mu_{t}=1$. Furthermore, with this scaling $\mu_{t}$ is a measure of how far away from the target value the process mean is relative to the distance between the target value and the upper specification limit, while $\sigma_{t}$ measures how large the process standard deviation $\sigma$ is relative to the distance between the target value and the upper specification limit. This is a convenient interpretation of closeness to target as well as spread. 
The contour curves are obtained by rewriting the indices in (6) and (7) as functions of $\mu_{t}$ and $\sigma_{t}$, solving the equations $C_{p u}(u, v)=k$ with respect to $\sigma_{t}$, and then plotting $\sigma_{t}$ as a function of $\mu_{t}$.

We easily find that $C_{p a u}(u, v)=k$, for $k>(1-u) /(3 \sqrt{v})$, is equivalent to

$$
\sigma_{t}=\left\{\begin{array}{l}
\frac{1-\mu_{t}-u\left|\mu_{t}\right|}{3 k}, \mu_{t}<\frac{1}{u+1} \text { for } v=0, \\
\sqrt{\frac{\left(1-\mu_{t}-u\left|\mu_{t}\right|\right)^{2}}{9 k^{2}}-v \mu_{t}^{2}}, \frac{-1}{u-1+3 k \sqrt{v}}<\mu_{t}<\frac{1}{u+1+3 k \sqrt{v}} \text { for } v>0 .
\end{array}\right.
$$

The case $k \leq(1-u) /(3 \sqrt{v})$ is studied in Appendix A for the sake of completeness, but it is not of any importance in practice.

Analogous we find that $C_{p v u}(u, v)=k$, for $k>(1-u) /(3 \sqrt{v})$, is equivalent to

$$
\sigma_{t}=\left\{\begin{array}{l}
\frac{1-\left|\mu_{t}\right|}{3 k},\left|\mu_{t}\right|<1 \text { for } u=1 \text { and } v=0, \\
\sqrt{\frac{\left(1-u\left|\mu_{t}\right|\right)^{2}}{9 k^{2}}-v \mu_{t}^{2}},\left|\mu_{t}\right|<\frac{1}{u+3 k \sqrt{v}} \text { for } v>0 .
\end{array}\right.
$$

As for $C_{p a u}(u, v)$, the case $k \leq(1-u) /(3 \sqrt{v})$ is studied in Appendix A.

Values of the process parameters $\mu$ and $\sigma$ which give $\left(\mu_{t}, \sigma_{t}\right)$-values inside the region bounded by the contour curves $C_{p u}(u, v)=k$, and the $\mu_{t}$-axis will give rise to a $C_{p u}(u, v)$-value larger than $k$, i.e. a capable process. We call this region the capability region. Furthermore, values of $\mu$ and $\sigma$ which give $\left(\mu_{t}, \sigma_{t}\right)$-values outside this region will give a $C_{p u}(u, v)$-value smaller than $k$, i.e. a non-capable process. Figures $1-2$ show some examples of capability regions when $k=4 / 3$.

Figure 1 illustrates the requirements the capability indices put on the process parameters regarding closeness to target and spread when $v=0$, and Figure 2 when $v>0$. In Figure 2 we see that although there is an upper limit only, the process mean $\mu$ cannot shift too far away to the left of the target value and still give rise to a capable process. This is due to the fact that the indices $C_{p u}(u, v)$ are sensitive to departures of the process mean from the target value for $v>0$.

In Figures $1-2$ it can be seen that the index $C_{p v u}(u, v)$ gives contour curves symmetrical around $\mu_{t}=0(\mu=T)$, while $C_{p a u}(u, v)$ does not. Furthermore, when $\mu_{t}>0(\mu>T)$ the capability region defined by $C_{p a u}(u, v)$ is smaller than the capability region defined by $C_{p v u}(u, v)$. The opposite holds for $\mu_{t}<0(\mu<T)$. This means that the index $C_{p a u}(u, v)$ is more sensitive to departures of the process mean from the target value if the process mean moves towards USL than if it moves in the opposite direction. The index $C_{p v u}(u, v)$, on the other hand, does not have this property.

The capability region for $C_{p a u}(0,0)$ is bounded by a straight line with negative slope and the $\mu_{t}$-axis. See Figure 1 (a). Hence the index $C_{p a u}(0,0)$ allows large process spread when the process mean is much smaller than USL. From Figure 1 we can also see that for 
$\mu_{t}>0$ and fixed value of the index, the capability region is smaller for $C_{p a u}(1,0)$ than for $C_{\text {pau }}(0,0)$. Furthermore, $C_{\text {pau }}(1,0)$ puts an upper bound on $\sigma_{t}$ equal to $1 /(3 k)$ when $\mu_{t}<$ $0(\mu<T)$. The index $C_{p v u}(1,0)$ defines a capability region bounded by two straight lines that form a triangle with the $\mu_{t}$-axis, which is the same capability region as of $C_{p k}$ in (1).

(a) $u=0$ and $v=0$

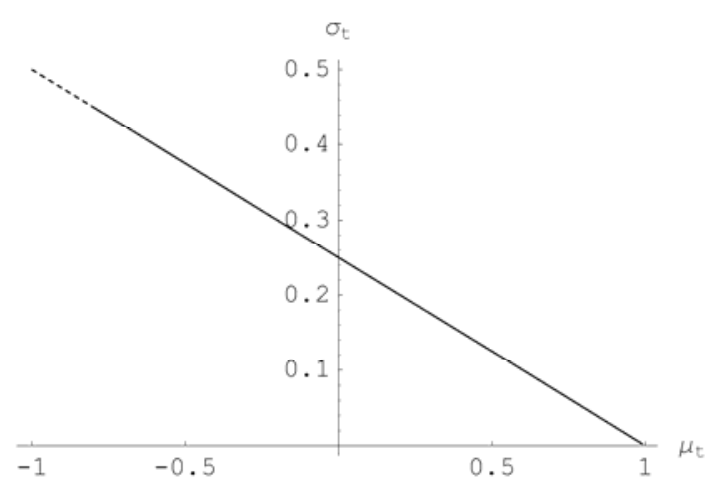

(b) $u=1$ and $v=0$

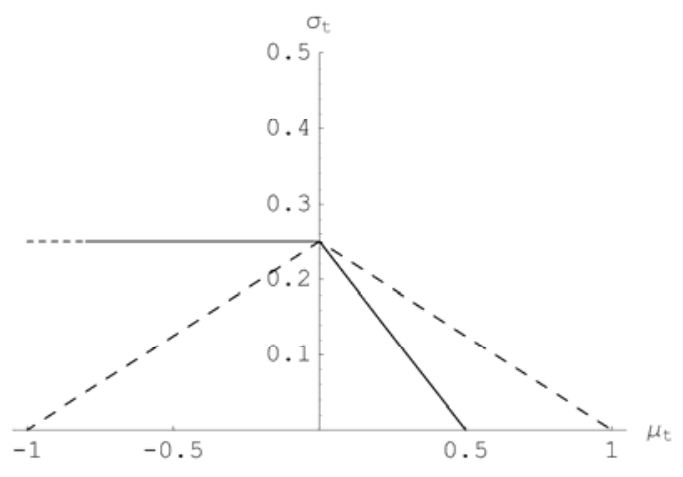

Figure 1. The contour curves for the process capability indices $C_{p a u}(u, v)=k$, and $C_{p v u}(u, v)=k$, when $k=4 / 3$. $C_{p a u}(u, v)$ corresponds to the continuous curve and $C_{p v u}(u, v)$ to the dashed curve. The region bounded by the contour curve and the $\mu_{t}$-axis is the corresponding capability region. Note that the index $C_{p v u}(u, v)$ is not defined for $(u, v)=(0,0)$.

(a) $u=0$ and $v=1$

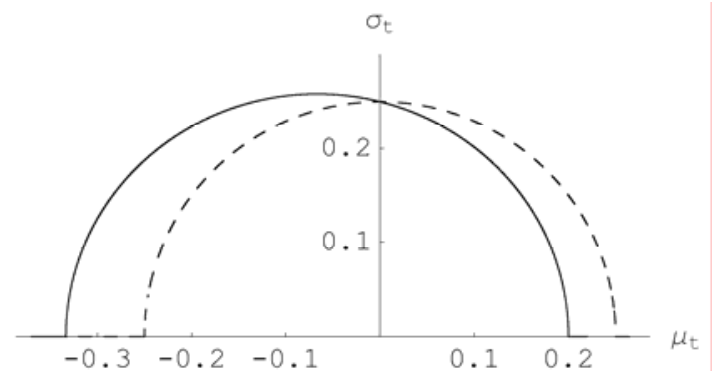

(c) $u=1$ and $v=1$

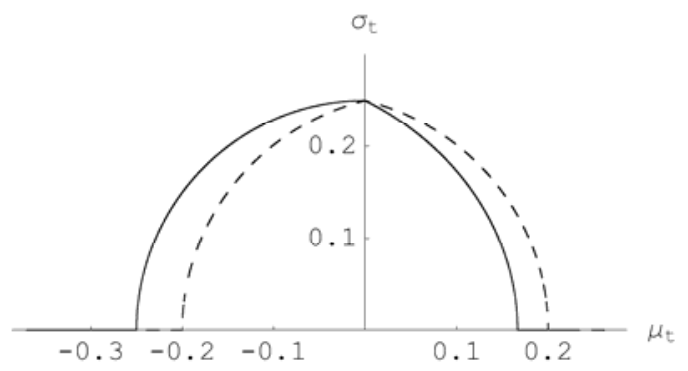

(b) $u=0$ and $v=4$

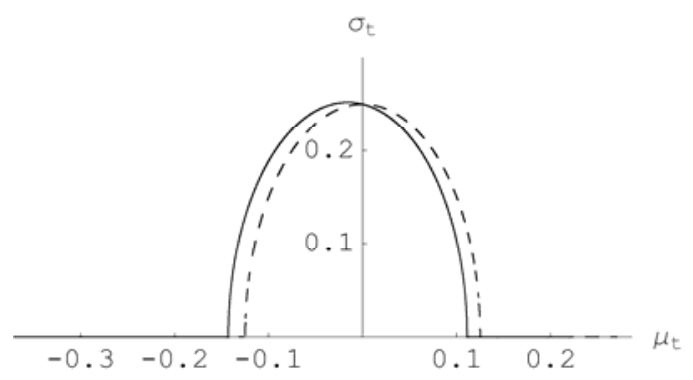

(d) $u=1$ and $v=4$

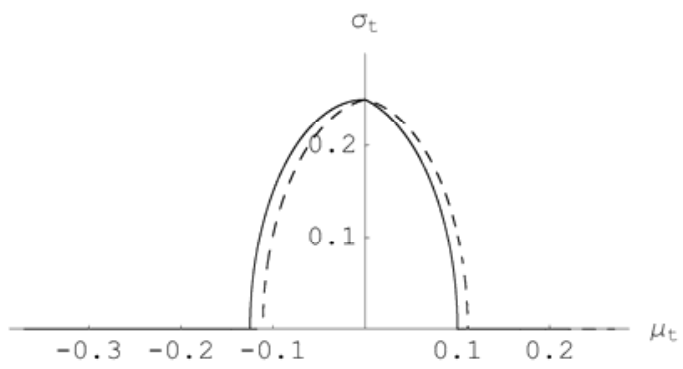

Figure 2. The contour curves for the process capability indices $C_{p a u}(u, v)=k$, and $C_{p v v}(u, v)=k$, when $k=4 / 3 . C_{p a u}(u, v)$ corresponds to the continuous curve and $C_{p v u}(u, v)$ to the dashed curve. The region bounded by the contour curve and the $\mu_{t}$-axis is the corresponding capability region. 
For $u=0$ and $v>0$ the capability region for $C_{p a u}(u, v)$ will be bounded by the $\mu_{t}$-axis and a semi-ellipse with the equation

$$
\frac{9 k^{2}\left(9 k^{2} v-1\right)}{9 k^{2}\left(9 k^{2} v-1\right)+1} \sigma_{t}^{2}+\frac{\left(9 k^{2} v-1\right)^{2}}{9 k^{2}\left(9 k^{2} v-1\right)+1}\left(\mu_{t}-\frac{1}{1-9 k^{2} v}\right)=1 \text {. }
$$

See Figure 2 (a)-(b). For the contour curve $C_{\text {pau }}(0, v)=k$, with $k>1 /(3 \sqrt{v})$, the maximum of $\sigma_{t}$ will be located to the left of the target value at

$$
\mu_{t}=\frac{1}{1-9 k^{2} v}
$$

with the maximum value

$$
\sigma_{t}=\sqrt{\frac{v}{9 k^{2} v-1}} .
$$

This means that for a given value of the index $C_{p a u}(0, v)$ for $v>0$, the process spread can be somewhat larger for $\mu$ in the vicinity but to the left of the target value compared to when $\mu=T$. However, in order to still give the same index value, the process spread then has to decrease if $\mu$ moves further away from the target value. This is due to the fact that $C_{p a u}(0, v)$ is sensitive to departures of the process mean from the target value for $v>0$.

For $u=1, v>0$ the contour curve $C_{p a u}(1, v)=k$ will have the maximum located at the target value, but with different absolute value of the gradient for $\mu<T$ and $\mu>T$, respectively. See Figure 2 (c). This means that the index allows, for a fixed value of $\sigma_{t}$, a larger deviation from target for $\mu_{t}<0(\mu<T)$ than for $\mu_{t}>0(\mu>T)$.

One easily finds that the contour curve of $C_{p v u}(0, v)$ is a semi-circle with a radius equal to $1 /\left(9 k^{2}\right)$ for $v=1$ and a semi-ellipse with equation

$$
9 k^{2} \sigma_{t}^{2}+9 k^{2} v \mu_{t}^{2}=1
$$

for $v>1$. See Figure 2 (a)-(b).

By using process capability plots, as exemplified by Figures 1-2, the restrictions that an index imposes on the process parameters can be easily seen, as well as the properties of the indices themselves. The process capability plot moves the focus from the formula of the index to the joint behaviour of the process parameters. Hence the process capability plots give new insights about the indices that are missing in Vännman [14].

The capability plots are also helpful when choosing an index. As an example, consider a situation with a manufacturing process producing units where the manufacturing cost increases with a decreasing value of the process mean $\mu$. Assume that there is a choice between the two indices $C_{p a u}(0,1)$ and $C_{p a u}(1,0)$. By making process capability plots, as in Figure 3, we immediately see that using the index $C_{\text {pau }}(0,1)$ the process will be claimed non-capable if the process mean gets too small. Hence this index will protect against increasing costs due to changes in the process mean. This will not be done by using the index $C_{p a u}(1,0)$. The same conclusion can be reached by studying the formulas for the indices. However, the plots show the properties more clearly. 


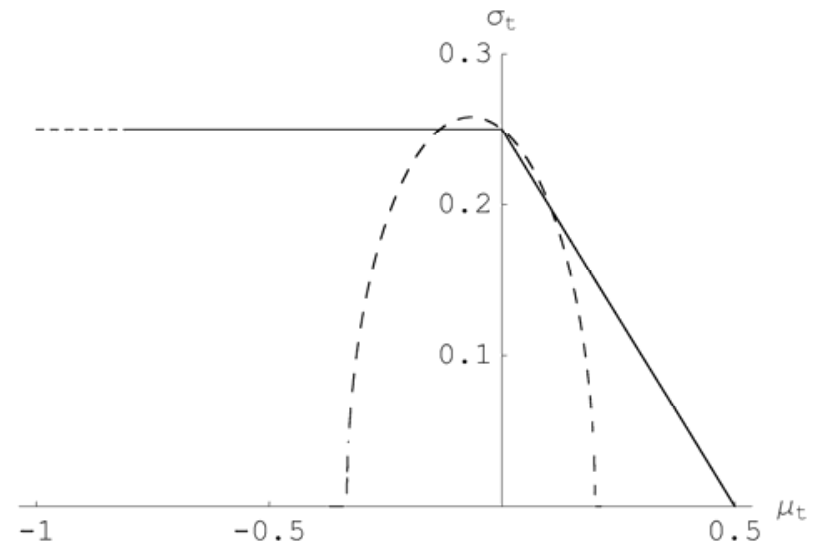

Figure 3 . The contour curves for the process capability indices $C_{p a u}(0,1)=4 / 3$, and $C_{p a u}(1,0)=4 / 3$. $C_{p a u}(0,1)$ corresponds to the dashed curve and $C_{p a u}(1,0)$ to the continuous curve.

\section{Estimated Process Capability Plots}

In most situations the process parameters $\mu$ and $\sigma$ are unknown and need to be estimated. Hence the obtained estimated indices have to be treated as random variables and their distributions have to be taken into account. We will now develop a graphical decision rule to be used for deciding whether a process can be considered capable or not at a given significance level, when $\mu$ and $\sigma$ are unknown. This rule is based on an estimated process capability plot, derived from sample statistics.

We treat the case when the studied characteristic, $X$, of the process is normally distributed with mean $\mu$ and variance $\sigma^{2}$ and the process is considered to be in statistical control. Let $X_{1}, X_{2}, \ldots, X_{n}$ be a random sample from the normal distribution measuring the studied characteristic $X$.

We will assume that a capability region has been defined by (9) or (10) for given values of $u, v$, and $k=k_{0}$, i.e. the studied process is defined to be capable if $C_{p u}(u, v)>k_{0}$. As in the previous section we consider the cases when $u=0,1$ and $v \geq 0$. To obtain an appropriate decision rule we consider a hypothesis test with the null hypothesis $H_{0}: C_{p u}(u, v) \leq k_{0}$ and the alternative hypothesis $H_{1}: C_{p u}(u, v)>k_{0}$. The test statistic is formed by estimating the mean $\mu$ by the sample mean and the variance $\sigma^{2}$ by its maximum likelihood estimator, i.e.

$$
\hat{\mu}=\bar{X}=\frac{1}{n} \sum_{i=1}^{n} X_{i} \quad \text { and } \quad \hat{\sigma}^{2}=\frac{1}{n} \sum_{i=1}^{n}\left(X_{i}-\bar{X}\right)^{2} .
$$

Hence, the test statistics are

$$
\hat{C}_{p a u}(u, v)=\frac{U S L-\hat{\mu}-u|\hat{\mu}-T|}{3 \sqrt{\hat{\sigma}^{2}+v(\hat{\mu}-T)^{2}}},
$$

and

$$
\hat{C}_{p v u}(u, v)=\frac{U S L-T-u|\hat{\mu}-T|}{3 \sqrt{\hat{\sigma}^{2}+v(\hat{\mu}-T)^{2}}} .
$$


The notation $\hat{C}_{p u}(u, v)$ will be used to denote either of the two estimators in (16) and (17), when it is not of interest to specify one of them.

By using the distribution of $\hat{C}_{p u}(u, v)$, which has been derived by Vännman [14], the critical value $c_{\alpha}$ of the test can be determined. The null hypothesis will be rejected whenever $\hat{C}_{p u}(u, v)>c_{\alpha}$, where the constant $c_{\alpha}$ is determined so that the significance level of the test is $\alpha$. The decision rule to be used is then that, for given values of $\alpha$ and $n$, the process will be considered capable if $\hat{C}_{p u}(u, v)>c_{\alpha}$. In the next section we will describe in detail how to obtain the critical value $c_{\alpha}$.

Now we can illustrate the decision rule in a plot corresponding to the process capability plot, described in the previous section, to obtain an estimated process capability plot. To do so we introduce the notation

$$
\hat{\mu}_{t}=\frac{\hat{\mu}-T}{U S L-T}=\frac{\bar{X}-T}{U S L-T}
$$

and

$$
\hat{\sigma}_{t}=\frac{\sqrt{\hat{\sigma}^{2}}}{U S L-T}=\frac{\hat{\sigma}}{U S L-T}=\frac{1}{U S L-T} \sqrt{\frac{1}{n} \sum_{i=1}^{n}\left(X_{i}-\bar{X}\right)^{2}},
$$

for the estimators of $\mu_{t}$ and $\sigma_{t}$. respectively. We will then make a contour plot of the estimated index $\hat{C}_{p u}(u, v)$, when $\hat{C}_{p u}(u, v)=c_{\alpha}$, as a function of $\hat{\mu}_{t}$ and $\hat{\sigma}_{t}$. This plot is called the estimated process capability plot. Note that we will always have $c_{\alpha}>k_{0} \geq 1$ since the smallest value of $k_{0}$ to be used is 1 .

As in the previous section we easily find the following expression to make the estimated process capability plot, if it is based on $\hat{C}_{p a u}(u, v)=c_{\alpha}$

$$
\hat{\sigma}_{t}=\left\{\begin{array}{l}
\frac{1-\hat{\mu}_{t}-u\left|\hat{\mu}_{t}\right|}{3 c_{\alpha}}, \hat{\mu}_{t}<\frac{1}{u+1} \text { for } v=0, \\
\sqrt{\frac{\left(1-\hat{\mu}_{t}-u\left|\hat{\mu}_{t}\right|\right)^{2}}{9 c_{\alpha}^{2}}-v \hat{\mu}_{t}^{2}}, \frac{-1}{u-1+3 c_{\alpha} \sqrt{v}}<\hat{\mu}_{t}<\frac{1}{u+1+3 c_{\alpha} \sqrt{v}} \text { for } v>0 .
\end{array}\right.
$$
we get

Correspondingly, if the estimated process capability plot is based on $\hat{C}_{p v u}(u, v)=c_{\alpha}$

$$
\hat{\sigma}_{t}=\left\{\begin{array}{l}
\frac{1-\left|\hat{\mu}_{t}\right|}{3 c_{\alpha}},\left|\hat{\mu}_{t}\right|<1 \text { for } u=1, v=0, \\
\sqrt{\frac{\left(1-u\left|\hat{\mu}_{t}\right|\right)^{2}}{9 c_{\alpha}^{2}}-v \hat{\mu}_{t}^{2}},\left|\hat{\mu}_{t}\right|<\frac{1}{u+3 c_{\alpha} \sqrt{v}} \text { for } v>0 .
\end{array}\right.
$$

We can now use the estimated process capability plot to define an estimated capability region in a way corresponding to using the process capability plot. We then consider a process capable, at significance level $\alpha$, if the point with coordinates $\left(\hat{\mu}_{t}, \hat{\sigma}_{t}\right)$ falls inside this estimated capability region.

As an example assume that we have decided to use an index in the class $C_{p a u}(u, v)$ in 
(6) and choose $u=0, v=1$, and $k_{0}=4 / 3$ to define our capability region. See Figure 2 (a). Furthermore, we have a sample of size $n=80$ and the significance level $\alpha=0.05$. Then we find $c_{0.05}=1.5703$ using the cumulative distribution function in Vännman [14]. (See Section 4 for details.) To find out whether the studied process can be considered capable or not we make the estimated process capability plot in Figure 4 (a). Then we calculate the observed values of $\hat{\mu}_{t}$ and $\hat{\sigma}_{t}$ defined in (18), and (19) respectively, and plot the coordinates $\left(\hat{\mu}_{t}, \hat{\sigma}_{t}\right)$ in the estimated process capability plot in Figure 4 (a). If the observed value of $\left(\hat{\mu}_{t}, \hat{\sigma}_{t}\right)$ falls inside the estimated capability region defined by $\hat{C}_{\text {pau }}(0,1)=1.5703$ then the process will be considered capable. Otherwise it will not be considered capable. Hence, instead of calculating the estimated capability index in (16) and compare it with $c_{a}$, we use a graphical method to make the decision. Figure $4(\mathrm{~b})$ illustrates the estimated capability region if we use $C_{p v u}(0,1)$ defined in (10) with $k_{0}=4 / 3$ to define our capability region and have a sample of size $n=80$ with the significance level $\alpha=0.05$. In this case we get the critical value $c_{0.05}=1.5346$. (See Section 4 for details.) Comparing Figure 4 with Figure 2 (a) we see that the estimated capability region is smaller than the corresponding theoretical capability region.

In Figure 4 we have also added an assumed estimated point $\left(\hat{\mu}_{t}, \hat{\sigma}_{t}\right)=(0.13,0.15)$ to illustrate the conclusions that can be drawn. When using the index $C_{p v u}(0,1)$ to define process capability, we conclude from Figure 4 (b) that the process can be claimed capable at $5 \%$ significance level, since the point is inside the estimated capability region. However, when using the index $C_{p a u}(0,1)$ we see from Figure 4 (a) that the process cannot be claimed capable at $5 \%$ significance level, since the point is outside the estimated capability region. From Figure 4 (a) we can also see how to improve the process. Either the process mean can be adjusted so that it is closer to the target value or the process spread need to be minimized, or a combination of both. All this information we get instantly by looking at the plot in Figure 4 (a).

Note also the differences between the two indices $C_{p a u}(0,1)$ and $C_{p v u}(0,1)$. The index $C_{p a u}(0,1)$ is more sensitive to departures of the process mean from the target value, when the process mean shifts towards USL. This is obvious from Figure 4 since the estimated capability region for $\hat{\mu}_{t}>0$ is smaller in Figure 4 (a) compared to Figure 4 (b). This is also the reason why a different conclusion is drawn from Figure 4 (a) than from Figure 4 (b).

(a) $\hat{C}_{p a u}(0,1)=c_{0.05}=1.5703$

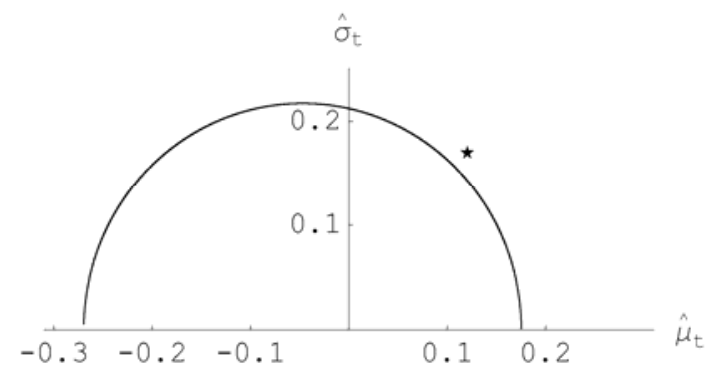

(b) $\hat{C}_{p v u}(0,1)=c_{0.05}=1.5346$

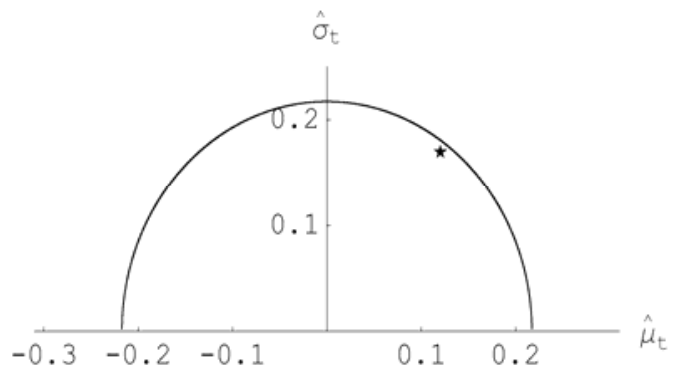

Figure 4. (a) The estimated capability region bounded by the contour curve defined by $\hat{C}_{p a u}(0,1)=1.5703$ and the $\hat{\mu}_{t}$-axis, when $n=80$. (b) The estimated capability region bounded by the contour curve de150fined by $\hat{C}_{p v u}(0,1)=1.5346$ and the $\hat{\mu}_{t}$-axis, when $n$ $=80$. The process can be deemed capable in (b) at $5 \%$ significance level but not in (a). 


\section{Significance Level and Power Determination}

We will now show how to obtain the critical value $c_{\alpha}$ to be used in the estimated capability plot described above, when the capability region has been defined by (9) or (10) for given values of $u, v$, and $k=k_{0}$, i.e. the studied process is defined to be capable if $C_{p u}(u, v)>k_{0}$. We consider the hypothesis test described in Section 3, where the null hypothesis is rejected when $\hat{C}_{p u}(u, v)>c_{\alpha}$.

In order to find the critical value $c_{\alpha}$ we have to be able to calculate $P\left(\hat{C}_{p u}(u, v)>c_{\alpha}\right)$, where only values of $c_{\alpha}>1$ and the cases $u=0,1$ are of interest. Let $F_{\xi}$ denote the cumulative distribution function of $\xi$, where $\xi$ is distributed according to a central $\chi^{2}$-distribution with $n-1$ degrees of freedom, and let $f_{\eta}$ denote the probability density function of $\eta$, where $\eta$ is distributed according to the standardized normal distribution $N(0,1)$. From formulas (19) and (20) in Vännman [14] we get for the estimated index $\hat{C}_{p a u}(u, v)$, when $u=0,1, v \geq 0$, that

$$
P\left(\hat{C}_{p a u}(u, v)>x\right)=\left\{\begin{array}{l}
\int_{-\infty}^{K /(u+1)} F_{\xi}\left(\frac{(K-t-u|t|)^{2}}{9 x^{2}}-v t^{2}\right) f_{\eta}(t-g) \mathrm{d} t, x>0 \text { for } v=0 \\
\int_{I_{L}}^{I_{U}} F_{\xi}\left(\frac{(K-t-u|t|)^{2}}{9 x^{2}}-v t^{2}\right) f_{\eta}(t-g) \mathrm{d} t, x>\frac{1-u}{3 \sqrt{v}} \text { for } v>0
\end{array},\right.
$$

where

$$
\begin{gathered}
K=\frac{(U S L-T) \sqrt{n}}{\sigma}=\frac{\sqrt{n}}{\sigma_{t}} \text { and } g=\frac{(\mu-T) \sqrt{n}}{\sigma}=\frac{\mu_{t} \sqrt{n}}{\sigma_{t}}, \\
I_{L}=\frac{-K}{u-1+3 x \sqrt{v}} \text { and } I_{U}=\frac{K}{u+1+3 x \sqrt{v}} .
\end{gathered}
$$

From formula (35) in Vännman [14] we find the corresponding probability for the index $\hat{C}_{p v u}(u, v)$, when $u=0,1, v \geq 0$, but $(u, v) \neq(0,0)$, to be

$$
P\left(\hat{C}_{p v u}(u, v)>x\right)=\int_{0}^{K /(u+3 x \sqrt{v})} F_{\xi}\left(\frac{(K-u t)^{2}}{9 x^{2}}-v t^{2}\right)\left(f_{\eta}(-t-g)+f_{\eta}(t-g)\right) \mathrm{d} t, x>0 .
$$

Consider first the case when $(u, v)=(0,0)$. This represents a special case, where the probability $P\left(\hat{C}_{p a u}(0,0)>x\right)$ in (22) depends on $\mu$ and $\sigma$ solely through the index $C_{p a u}(0,0)$. This can be seen by rewriting the integral in (22) using the simple transformation $y=K-t$. Then we obtain

$$
P\left(\hat{C}_{p a u}(0,0)>x\right)=\int_{0}^{\infty} F_{\xi}\left(\frac{y^{2}}{9 x^{2}}\right) f_{\eta}\left(3 \sqrt{n} C_{p a u}(0,0)-y\right) \mathrm{d} y, x>0 .
$$

Note that $C_{p a u}(0,0)=C_{P U}$ in (3). The distribution of the estimator of $C_{P U}$, when $\sigma^{2}$ is estimated by the sample variance $s^{2}$, has been derived by Chou and Owen [3]. Adjusting for the maximum likelihood estimator of $\sigma^{2}$ their result implies that $\hat{C}_{p a u}(0,0)$ is distributed as $(3 \sqrt{n-1})^{-1} t_{n-1}(\lambda)$, where $t_{n-1}(\lambda)$ is a non-central $t$-distribution with $n-1$ degrees of freedom and non-centrality parameter $\lambda=3 \sqrt{n} C_{P U}$. This result is 
equivalent to the result in (26). However, in (26) no non-central distribution is involved.

In the case when $(u, v)=(0,0)$ the probability in (22) depends on $\mu$ and $\sigma$ solely through the index $C_{p a u}(0,0)$. But when $(u, v) \neq(0,0)$ this is not the case, i.e. the probability $P\left(\hat{C}_{p a u}(u, v)>x\right)$ depends on $\mu$ and $\sigma$, but not solely through $C_{p a u}(u, v)$. This means that for a given value of $C_{p a u}(u, v)$ there is not a unique probability $P\left(\hat{C}_{p a u}(u, v)>x\right)$, when $(u, v) \neq(0,0)$. To calculate this probability we need given values of both $\mu$ and $\sigma$. The same is true for $P\left(\hat{C}_{p v u}(u, v)>x\right)$. Examples of how the probability $P\left(\hat{C}_{p a u}(u, v)>x\right)$ may vary for a given index value are shown in Figures 5 (a) and 6 (a). Since our null hypothesis $H_{0}: C_{p u}(u, v) \leq k_{0}$ is composite, for a significance level $\alpha$, the critical value $c_{\alpha}$ is defined as the unique smallest $c$ for which $\alpha(c) \leq \alpha$, where

$$
\alpha(c)=\sup \left\{P\left(\hat{C}_{p u}(u, v)>c\right):(\mu, \sigma) \text { such that } C_{p u}(u, v) \leq k_{0}\right\} .
$$

See, e.g. Bickel and Doksum [1], p. 217. To obtain $\alpha(c)$ we have to investigate $P\left(\hat{C}_{p u}(u, v)>c\right)$ for all values of $C_{p u}(u, v) \leq k_{0}$, i.e. for all values of $\left(\mu_{t}, \sigma_{t}\right)$ outside the capability region defined by the process capability plot for $C_{p u}(u, v)=k_{0}$, c.f. Figure 2 . It is reasonable to assume that the supremum in (27) will be found along the contour curve defined by $C_{p u}(u, v)=k_{0}$. Numerical investigations support this assumption. Hence we assume that we can reduce the null hypothesis $H_{0}: C_{p u}(u, v) \leq k_{0}$ to the hypothesis $H_{0}: C_{p u}(u, v)=k_{0}$, which still is a composite hypothesis when $(u, v) \neq(0,0)$. This is seen by studying the probability $P\left(\hat{C}_{p u}(u, v)>x \mid C_{p u}(u, v)=k_{0}\right)$, which depends on one of the process parameters. This means that $P\left(C_{p u}(u, v)>x \mid C_{p u}(u, v)=k_{0}\right)$ will vary depending on where along the contour curve $C_{p u}(u, v)=k_{0}$ the point $\left(\mu_{t}, \sigma_{t}\right)$ is located, c.f. Figures 5 (a) and 6 (a). Hence we have to explore $P\left(\hat{C}_{p u}(u, v)>c\right)$ for all points $\left(\mu_{t}, \sigma_{t}\right)$ on the contour curve $C_{p u}(u, v)=k_{0}$. To derive $c_{\alpha}$ analytically, for arbitrary values of $(u, v) \neq(0,0)$, does not seem feasible. Instead it has to be derived numerically for given values of $n$ and $\alpha$. In Appendix B the MATLAB code for obtaining the critical value $c_{\alpha}$, for given values of $u, v$, $n, \alpha$, and $k_{0}$, is given.

For the index $C_{p v u}(0,1)$ there is an analytic expression for the critical value $c_{\alpha}=c_{C_{p v u}(0,1), \alpha}$. The index $C_{p v u}(0,1)$ is the same as the index $C_{p m}$ in (2), since for the case of two-sided specification limits with the target value as midpoint we have USL - LSL = $U S L-T$. Furthermore, the cumulative distribution function of $\hat{C}_{p v u}(0,1)$ can easily be shown to be the same as the cumulative distribution function of the estimator of $C_{p m}$ as given in Vännman and Hubele [18]. Following the same reasoning as when Hubele and Vännman [6] obtain their formula (26), we find that

$$
c_{C_{p v u}(0,1), \alpha}=k_{0} \sqrt{\frac{n}{\chi_{\alpha, n}^{2}}},
$$

Using the code in Appendix B the critical values for the indices in Tables 1-2 are derived.

It can be noted from Tables 1 and 2 that the critical values are the same for the two indices $C_{p a u}(0,0)$ and $C_{p v u}(1,0)$. This follows from the fact that for the index $C_{p v u}(1,0)$ the supremum in (27) is obtained when $\mu_{t} \rightarrow 1$. Furthermore, when $\mu_{t} \rightarrow 1$, we have that $P\left(\hat{C}_{p v u}(1,0)>x \mid C_{p v u}(1,0)=k\right)$ equals the probability given in (26). The differences for two cases when $n$ is small are due to the numerical approximations in the algorithm. 
We will now return to the two examples considered in Section 3, where $k_{0}=4 / 3, n=$ 80 , and $\alpha=0.05$. Using the index $C_{p a u}(0,1)$ we get the critical value $c_{0.05}=1.5703$ from Table 2. Hence the process will be considered capable if the estimated index $\hat{C}_{p a u}(0,1)>1.5703$. The corresponding estimated capability region is plotted in Figure 4 (a). The probability $P\left(\hat{C}_{\text {pau }}(0,1)>1.5703\right)$ is a function of $\mu_{t}$ and attains it maximum 0.05 for $\mu_{t}=0.0991$. In Figure 5 (a) $P\left(\hat{C}_{p a u}(0,1)>1.5703\right)$ is plotted as a function of $\mu_{t}$, when the parameters $\left(\mu_{t}, \sigma_{t}\right)$ move along the contour curve $C_{p a u}(0,1)=4 / 3$ and we can see how this probability changes. The reason for this change is that the distribution of $\hat{C}_{p a u}(0,1)$ will differ considerably depending on where the point $\left(\mu_{t}, \sigma_{t}\right)$ is located along the contour curve $C_{p a u}(0,1)=4 / 3$. To illustrate this the probability density function of $\hat{C}_{\text {pau }}(0,1)$ is plotted in Figure 5 (b) for different choices of $\left(\mu_{t}, \sigma_{t}\right)$ such that $C_{p a u}(0,1)=4 / 3$. The probability density function of $\hat{C}_{p a u}(0,1)$ is obtained by taking the derivate of the cumulative distribution function in (22).

Table 1 . The critical value $c_{\alpha}$, when $\alpha=0.01$ and $n=30,50,80,100,150,200$, for the indices $C_{p a u}(0,0), C_{p a u}(0,1), C_{p a u}(0,4), C_{p v u}(1,0)$, and $C_{p v u}(1,4)$.

\begin{tabular}{|c|c|c|c|c|c|}
\hline Index & $C_{\text {pau }}(0,0)$ & $C_{\text {pau }}(0,1)$ & $C_{\text {pau }}(0,4)$ & $C_{p v u}(1,0)$ & $C_{p v u}(1,4)$ \\
\hline$n=30$ & 1.9611 & 1.9796 & 2.2793 & 1.9611 & 2.2788 \\
\hline$n=50$ & 1.7728 & 1.7971 & 2.0192 & 1.7742 & 2.0191 \\
\hline$n=80$ & 1.6582 & 1.6815 & 1.8504 & 1.6582 & 1.8504 \\
\hline$n=100$ & 1.6165 & 1.6385 & 1.7872 & 1.6165 & 1.7871 \\
\hline$n=150$ & 1.5557 & 1.5751 & 1.6931 & 1.5557 & 1.6931 \\
\hline$n=200$ & 1.5216 & 1.5390 & 1.6395 & 1.5216 & 1.6395 \\
\hline
\end{tabular}

Table 2. The critical value $c$, when $\alpha=0.05$ and $\mathrm{n}=30,50,80,100,150,200$, for the indices $C_{p a u}(0,0), C_{p a u}(0,1), C_{p a u}(0,4), C_{p v u}(1,0)$, and $C_{p v u}(1,4)$.

\begin{tabular}{|c|c|c|c|c|c|}
\hline Index & $C_{p a u}(0,0)$ & $C_{p a u}(0,1)$ & $C_{p a u}(0,4)$ & $C_{p v u}(1,0)$ & $C_{p v u}(1,4)$ \\
\hline$n=30$ & 1.7535 & 1.7600 & 1.9516 & 1.7551 & 1.9514 \\
\hline$n=50$ & 1.6324 & 1.6452 & 1.7899 & 1.6324 & 1.7899 \\
\hline$n=80$ & 1.5566 & 1.5703 & 1.6820 & 1.5566 & 1.6820 \\
\hline$n=100$ & 1.5287 & 1.5420 & 1.6409 & 1.5287 & 1.6409 \\
\hline$n=150$ & 1.4876 & 1.4998 & 1.5791 & 1.4876 & 1.5791 \\
\hline$n=200$ & 1.4643 & 1.4756 & 1.5435 & 1.4643 & 1.5435 \\
\hline
\end{tabular}

Using the index $C_{p v u}(0,1)$, we obtain the critical value $c_{0.05}=1.5346$ from formula (28) with $k_{0}=4 / 3, n=80$, and $\alpha=0.05$. Hence the process will be considered capable if $\hat{C}_{p v u}(0,1)>1.5346$. The corresponding estimated capability region is plotted in Figure $4(\mathrm{~b})$. In Figure 6 (a) $P\left(\hat{C}_{p v u}(0,1)>1.5346\right)$ is plotted as a function of $\mu_{t}$, when the parameters $\left(\mu_{t}, \sigma_{t}\right)$ move along the contour curve $C_{p v u}(0,1)=4 / 3$ and we can see how this probability varies. The probability $P\left(\hat{C}_{p v u}(0,1)>1.5346\right)$ attains it maximum 0.05 for $\mu_{t}=0$. In Figure $6(\mathrm{~b})$ the probability density function of $\hat{C}_{p v u}(0,1)$ is shown for different choices of $\left(\mu_{t}, \sigma_{t}\right)$ such that $C_{p v u}(0,1)=4 / 3$. This illustrates the varying values obtained for the probability $P\left(\hat{C}_{p v u}(0,1)>1.5346\right)$ given that $C_{p v u}(0,1)=4 / 3$.

For a given value of $C_{p u}(u, v)=k_{1}>k_{0}$, the power, as a function of $\mu_{t}$ can be calculated for each test based on $\hat{C}_{p a u}(u, v)$ and $\hat{C}_{p v u}(u, v)$, using (22) and (25), respectively. In this way a suitable sample size can be determined to ensure that the test will detect, with large probability, if the process gives rise to values of $\mu_{t}$ and $\sigma_{t}$ in the capability region. 
In Figure 7 we illustrate the power, as function of $\mu_{t}$, for $C_{\text {pau }}(0,1)=1.6,1.7,1.8$ and $C_{p v u}(0,1)=1.6,1.7,1.8$, respectively, when the parameters $\left(\mu_{t}, \sigma_{t}\right)$ move along the corresponding contour curve.

Note that the estimated capability regions of the two indices $C_{p a u}(0,1)$ and $C_{p v u}(0,1)$ differ. See Figure 4. Furthermore, $C_{p a u}(0,1)=1.6$ give rise to a different set of $\left(\mu_{t}, \sigma_{t}\right)$-values compared to $C_{p v u}(0,1)=1.6$. This has to be taken into consideration if comparing the power in Figures 7 (a) and (b).

(a) $P\left(\hat{C}_{p a u}(0,1)>1.5703 \mid C_{p a u}(0,1)=4 / 3\right)$

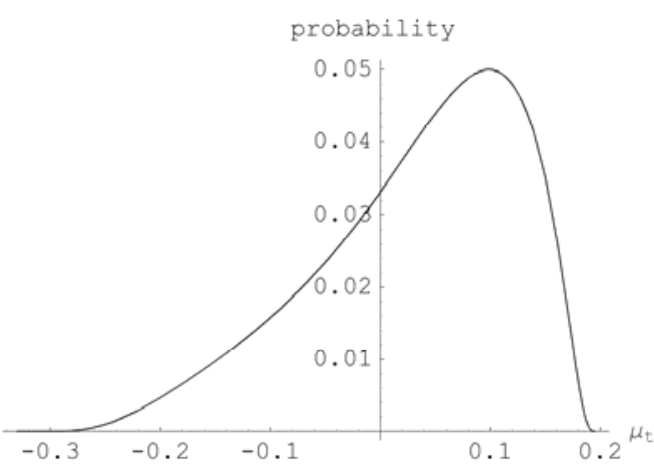

(b) The probability density function of $\hat{C}_{p a u}(0,1)$

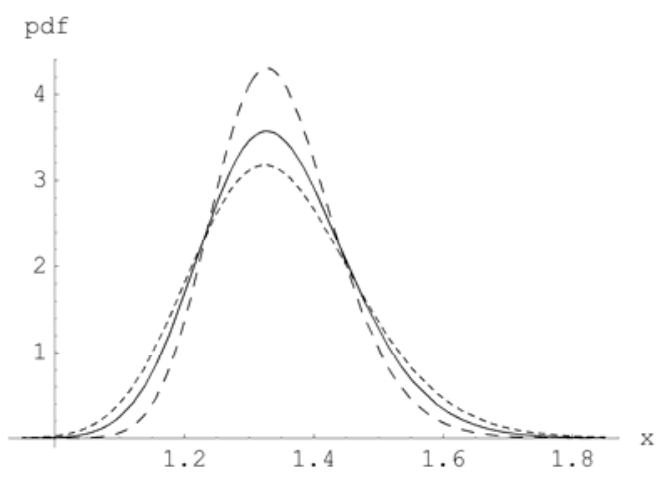

Figure 5. (a) The probability that the process is considered capable, i.e. $P\left(\hat{C}_{p a u}(0,1)>1.5703\right)$, given that $C_{p a u}(0,1)=4 / 3$, for $n=80$, as a function of $\mu_{t}$. (b) The probability density function of $C_{\text {pau }}(0,1)$ for $n=80$ given different values of $\left(\mu_{t}, \sigma_{t}\right)$ along the contour curve $C_{p a n}(0,1)=4 / 3$. From the top the dashed curve corresponds to $\mu_{t}=-0.2$, the continuous curve corresponds to $\mu_{t}=0$, and the dotted curve corresponds to $\mu_{t}=0.1$.

(a) $P\left(\hat{C}_{p v u}(0,1)>1.5346 \mid C_{p v u}(0,1)=4 / 3\right)$

(b) The probability density function of

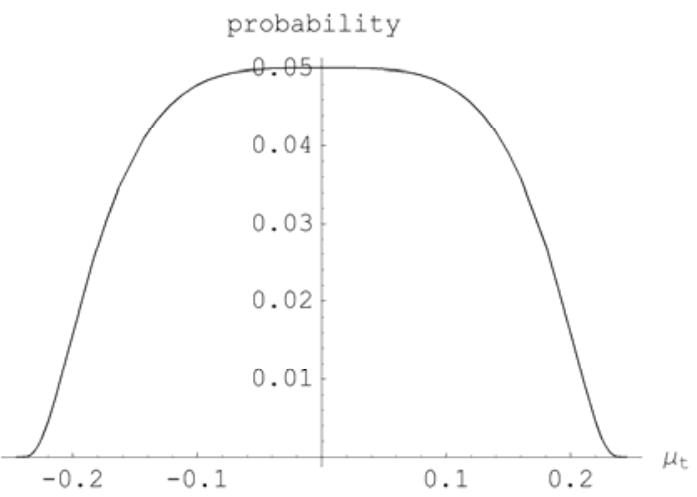
$\hat{C}_{p v u}(0,1)$

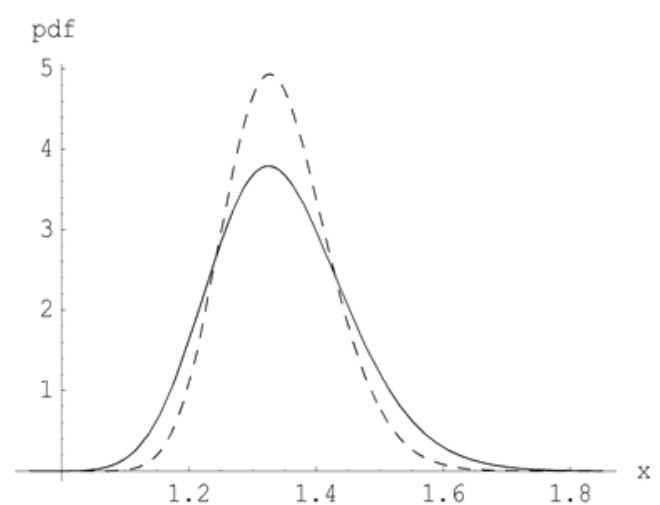

Figure 6.(a) The probability that the process is considered capable, i.e. $P\left(\hat{C}_{p v u}(0,1)>1.5346\right)$ given that $C_{p v u}(0,1)=4 / 3$, for $n=80$. (b) The probability density function of $\hat{C}_{p v u}(0,1)$ for $n=80$ given different values of $\left(\mu_{t}, \sigma_{t}\right)$ along the contour curve $C_{p v u}(0,1)=4 / 3$. From the top the dashed curve corresponds to $\mu_{t}=0.2$ and the continuous curve corresponds to $\mu_{t}=0$. 
(a) $C_{p a u}(0,1)$

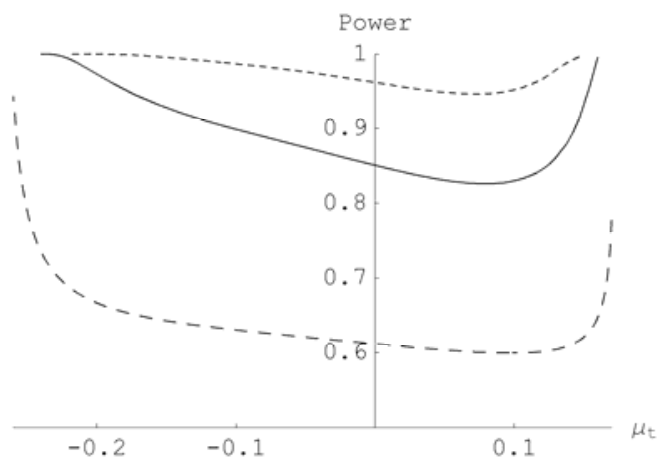

(b) $C_{p v u}(0,1)$

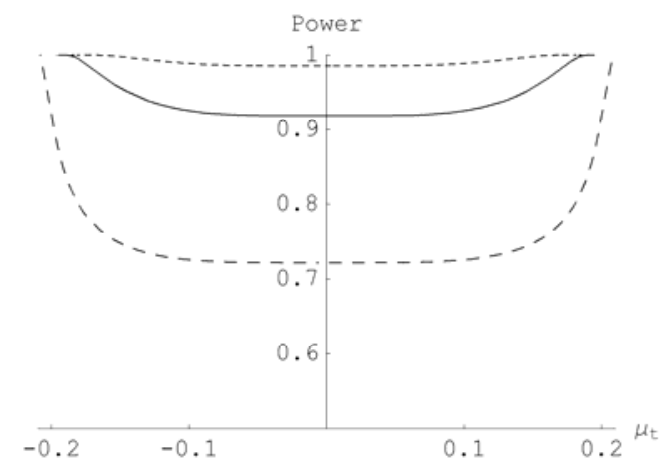

Figure 7. (a) The power for $C_{p a u}(0,1)=1.6,1.7,1.8$, when $H_{0}: C_{p a u}(0,1)=4 / 3$, for $n=80$. (b) The power for $C_{p v u}^{p a u}(0,1)=1.6,1.7,1.8$, when $H_{0}: C_{p v u}(0,1)=4 / 3$, for $n=80$. From the top the dotted curve corresponds to $C_{p u}(0,1)=1.8$, the continuous curve corresponds to $C_{p u}(0,1)=1.7$, and the dashed curve corresponds to $C_{p u}(0,1)=1.6$.

The maximum likelihood estimator in (15), and not the sample variance $s^{2}$, was used above as an estimator of $\sigma^{2}$. One reason for this is that it simplifies the distribution of $\hat{C}_{p u}(u, v)$ somewhat. If instead the sample variance $s^{2}$ is used as an estimator of $\sigma^{2}$, when estimating $C_{p u}(u, v)$, we can carry out the same reasoning as above. We only have to utilize the following simple relationship in (30). Let $\tilde{C}_{p u}(u, v)$ denote the estimator of $C_{p u}(u, v)$ when using $s^{2}$ as estimator of $\sigma^{2}$. Then we have

$$
\tilde{C}_{p u}(u, v)=\sqrt{\frac{n-1}{n}} \cdot \hat{C}_{p u}\left(u, \frac{v(n-1)}{n}\right) .
$$

Using the result in (30) we can easily derive the distribution of $\tilde{C}_{p u}(u, v)$ from the distribution of $\hat{C}_{p u}(u, v)$.

\section{Examples}

As an illustration of the ideas presented above we will consider a practical example described by Pearn and Shu [13]. They studied the quiescent current in low-drop-out 3A linear regulators, where the upper specification limit, USL, was set to $650 \mu \mathrm{A}$. Based on a sample of size 80 they investigated if the process was capable with respect to this characteristic. From their sample data we get the observed values of $\hat{\mu}$ and $\hat{\sigma}$ as

$$
\hat{\mu}=398.650 \text { and } \hat{\sigma}=61.264
$$

Pearn and Shu [13] used the index $C_{P U}$ defined in (3) to study the capability and obtained a lower $95 \%$ confidence bound for $C_{P U}$. They showed that it is reasonable to regard the sample data as taken from a normal distribution.

The index $C_{P U}$ defined in (3) is equivalent to the index $C_{p a u}(0,0)$ and its capability region is plotted in Figure 1 (a). This is an index that is not based on a target value. To illustrate our reasoning in this article we will assume that there is a target value, $T$, and set $T=340 \mu \mathrm{A}$. As null hypothesis we will consider $H_{0}: C_{p u}(u, v) \leq 4 / 3$ with the alternative hypothesis $H_{1}: C_{p u}(u, v)>4 / 3$ and set the significance level $\alpha=0.05$. We will use the indices $C_{p a u}(0,1)$ and $C_{p v u}(0,1)$, which have been discussed in the previous section. We 
will also consider the index $C_{p v u}(1,4)$. This index was found by Vännman [14] to achieve large power, when the process is on target, and at the same time have high sensitivity against departures from the process mean. We will also compare the results with the index $C_{p a u}(0,0)=C_{P U}$.

We get the critical values $c_{0.05}$ from Table 2 and formula (28). Using these values we obtain estimated process capability plots according to (20) and (21). We then calculate the observed values of $\hat{\mu}_{t}$ and $\hat{\sigma}_{t}$ defined in (18) and (19), respectively, and find them to be

$$
\hat{\mu}_{t}=0.189 \text { and } \hat{\sigma}_{t}=0.198 \text {. }
$$

then the point with coordinates $(0.189,0.198)$ is plotted in the estimated process capability plot. The results for the four indices are found in Figure 8 (a)-(d).

From Figure 8 we can see that that process cannot be deemed capable at 5\% significance level irrespectively of which index we use as definition of a capable process. In each of the four estimated process capability plots the observed value of $\left(\hat{\mu}_{t}, \hat{\sigma}_{t}\right)$ falls outside the estimated capability region. We can also see instantly how the process needs to be changed in order to be considered as capable. This change will depend on which index is used for the definition of a capable process.

(a) $C_{p v u}(0,1)$

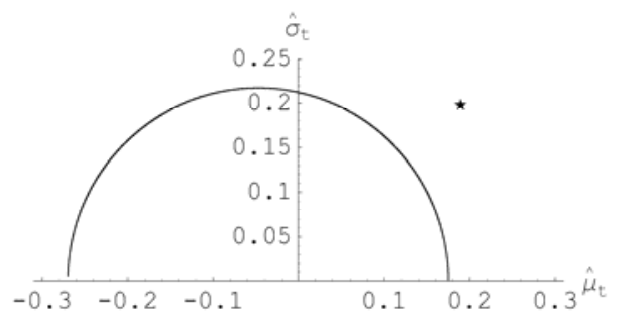

(c) $C_{p v u}(1,4)$

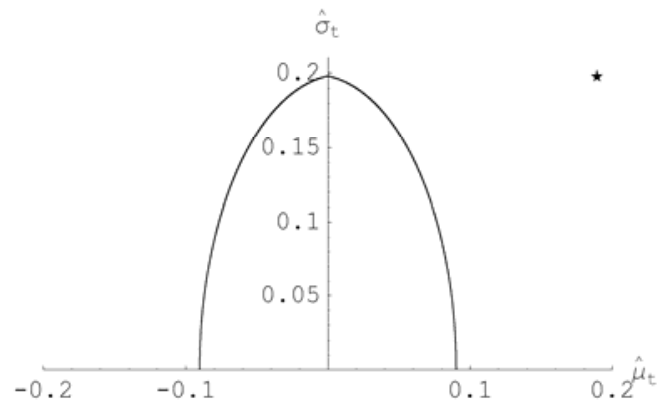

(b) $C_{p v u}(0,1)$

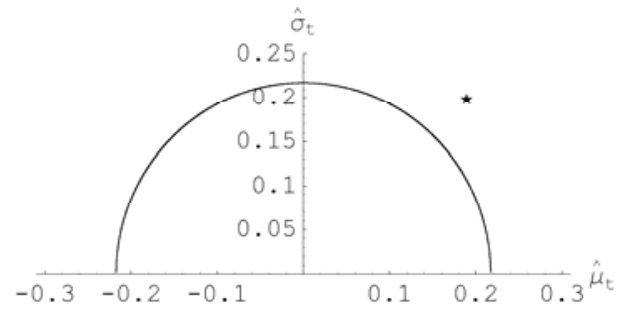

(d) $C_{p a u}(0,0)$

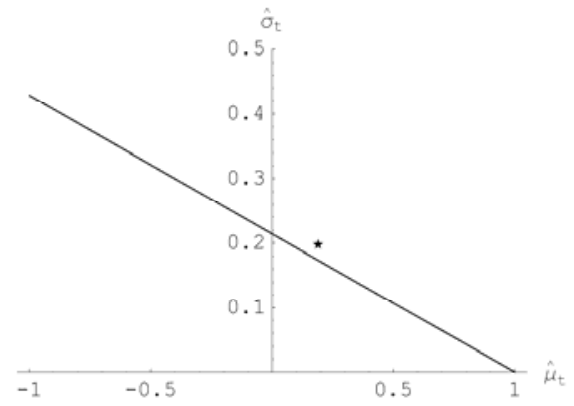

Figure 8 . The estimated process capability plots at $5 \%$ significance level, in (a) defined by $\hat{C}_{p a u}(0,1)=1.5703$; in (b) defined by $\hat{C}_{p v u}(0,1)=1.5346$; in (c) defined by $\hat{C}_{p v u}(1,4)=1.6820$; in (d) defined by $\hat{C}_{p a u}(0,0)=1.5566$. Each plot shows that the process cannot be considered capable at 5\% significance level.

If $C_{p a u}(0,1)$ is used, we can conclude from Figure $8(\mathrm{a})$, that a change in the process mean is needed so that it will be closer to the target value. We can also see that the process mean needs to be decreased with about $0.18 \cdot(U S L-T)=0.18 \cdot(650-340)=55.8 \mu \mathrm{A}$ if there is no change in the spread. Furthermore, decreasing the spread alone will not be sufficient to obtain a capable process. This will, however, be possible if the index $C_{p v u}(0,1)$ 
is used, which can be seen from Figure 8 (b). If the process mean is not changed but the process standard deviation is decreased to about $0.1 \cdot(U S L-T)=0.1 \cdot(650-340)=31 \mu \mathrm{A}$ the index $C_{p v u}(0,1)$ will claim the process as capable. If the process standard deviation is not changed there is a need for a decrease of the process mean with about $0.12 \cdot(U S L-T)=0.12 \cdot(650-340)=37.2 \mu \mathrm{A}$, which is less than for the index $C_{p v u}(0,1)$.

The difference in estimated capability regions when comparing Figure $8(a)$ and (b) is not as large as when comparing these two regions with the estimated capability region of the index $C_{p v u}(1,4)$. From Figure $8(\mathrm{c})$ we see that a change is needed both in the process mean and the spread in order to obtain a capable process based on the index $C_{p v u}(1,4)$. We see clearly that this index is very sensitive to deviations of the process mean from the target value. Such an index should be used when this sensitivity is important.

An index not sensitive to deviations of the process mean from the target value is $C_{p a u}(0,0)=C_{P U}$ in Figure $8(\mathrm{~d})$. We can see that if either the process mean is decreased with about $0.1 \cdot(U S L-T)=0.1 \cdot(650-340)=31 \mu \mathrm{A}$ or the process spread is decreased the same amount the process will be considered capable according to the definition based on $C_{p a u}(0,0)=C_{P U}$. Note that the scale in Figure 8 (d) differs from the scale used in Figures 8 (a)-(c). If all four estimated capability regions are plotted in one figure we can see more clearly the different requirements they put on the process parameters. See Figure 9.

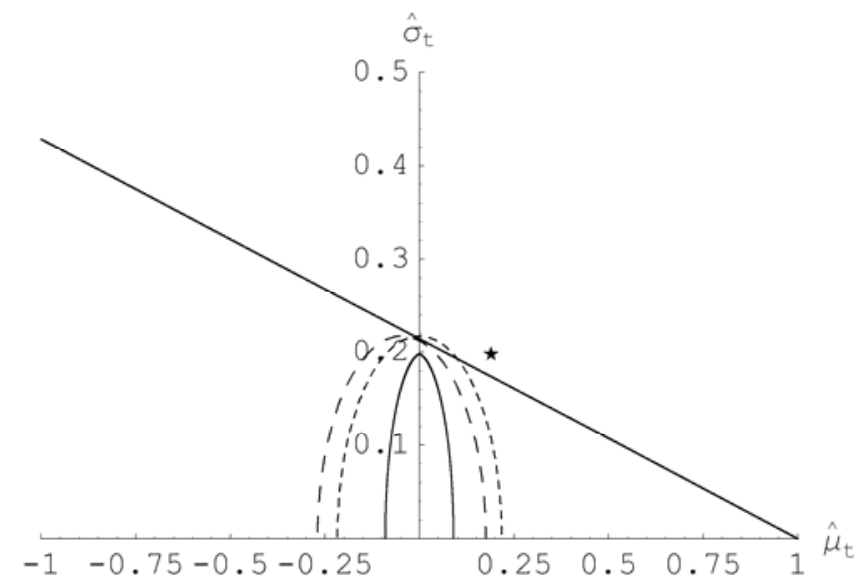

Figure 9. The estimated process capability plots from Figure 8 (a)-(d) joined together.

\section{Discussion and Concluding Remarks}

In this paper we present a graphical method to be used when deeming a process as capable or not at a given significance level for the case when the studied characteristic is normally distributed and specification limit is one-sided. This method is based on a test, which generalizes the previously presented test in Vännman [14], where the null hypothesis is that the process is on target and the capability index attains a given number. In the test studied here, the null hypothesis does not require that the process is on target. Hence, it takes into consideration that a given value of the capability index implies that the process parameters vary along a contour curve. This is illustrated in an efficient way by the process capability plots.

The graphical method discussed here is efficient in the respect that in one single plot we get visual information about not only the capability of the process at a given signifi- 
cance level but simultaneously also information about the location and spread of the studied characteristic. In the estimated process capability plot we can at a glance relate the deviation from target and the spread to each other and to the capability index in such a way that we are able to see whether a non-capability is caused by the fact that the process output is off target, or that the process spread is too large, or if the result is a combination of these two factors. Furthermore, we can easily see how large a change of the process parameters that is needed to obtain a capable process.

The suggested process capability plots are invariable, irrespective of the values of the specification limits. Hence we can monitor, in the same plot, several characteristics of a process and at the same time retain the information on the location and spread of the process. In this way more information is obtained on how to improve the process compared to using traditional capability indices alone.

When an upper specification USL exists it is not uncommon that the smallest possible value of the characteristic is 0 and this also is the best value to obtain. As an example consider a surface polishing process, where the surface should be as smooth as possible and ideally should have the smoothness values of 0 . It is not unusual in practice in such a situation to use a two-sided specification interval and put $L S L=0$. However, this will imply that when the smoothness values are close to zero the index value will be small, possibly indicating a non-capable process.

A better way to treat the described situation is to include a target value $T=0$ and apply any of the indices studied in this paper. If the smallest possible value of the studied characteristic is 0 then the process capability regions will be defined for values of $\mu_{t} \geq 0$ only. Using process capability plots for $\mu_{t} \geq 0$ we can chose an index with suitable sensitivity to departures from 0 .

If it is reasonable to assume that the studied characteristic is normally distributed the theory and the estimated capability plot described in this paper can be used. However, in a situation such as the one exemplified above, it is not unusual to find a skew distribution with a long tail towards large values instead of a normal distribution. This case has been considered by Vännman and Albing [17]. They propose a new index suitable for this special situation and suggest a decision procedure based on large sample properties.

Using the herein discussed plots, compared to using the capability index alone, we will, from one single plot, instantly get visual information simultaneously about the location and spread of the quality characteristic, as well as information about the capability of the process at a stated significance level. In this way, the proposed plots give clear directions for quality improvement. The capability indices were introduced to focus on the process variability and closeness to target and relate these quantities to the specification interval and the target value. We believe that the plots discussed here will do this in a more efficient way than the capability index alone. It is also well known that the visual impact of a plot is more efficient than numbers, such as estimates or confidence limits. Furthermore, with today's modern software the plots proposed here are easy to generate.

\section{Acknowledgements}

The authors are grateful for the financial support provided by the Swedish Research Council, project number 621-2002-3823. In addition, we appreciate the very thorough and thoughtful comments of three anonymous referees that have helped to improve and clarify our presentation. 


\section{References}

1. Bickel, P. J. and Doksum, K. A. (2001). Mathematical Statistics. Basic Ideas and Selected Topics, Volume I. Second Edition. Prentice Hall, Upper Saddle River, New Jersey 07458.

2. Chan, L. K., Cheng, S. W. and Spiring, F. A. (1988). A new measure of process capability: $C_{p m}$. Journal of Quality Technology, 20, 162-175.

3. Chou, Y. M. and Owen, D. B. (1989). On the distribution of the estimated process capability indices. Communications in Statistics-Theory and Methods, 18, 4549-60.

4. Deleryd, M. and Vännman, K. (1999). Process capability plots - a quality improvement tool. Quality and Reliability Engineering International, 15, 1-15.

5. Gunter, B. H. (1989). The use and abuse of $C_{p k}$ : Parts 1-4. Quality Progress, 22, January, 72-73; March, 108-109; May, 79-80; July, 86-87.

6. Hubele, N. F. and Vännman, K. (2004). The effect of pooled and un-pooled variance estimators on $C_{p m}$ when using subsamples. Journal of Quality Technology, 36, 207-222.

7. Kane, V. E. (1986). Process capability indices. Journal of Quality Technology, 18, 41-52.

8. Kotz, S. and Johnson, N. L. (1993). Process Capability Indices. Chapman \& Hall, London.

9. Kotz, S. and Johnson, N. L. (2002). Process capability indices - a review, 1992- 2000 with discussion. Journal of Quality Technology, 34, 2-53.

10. Kotz, S. and Lovelace, C. R. (1998). Introduction to Process Capability Indices: Theory and Practice. Arnold, London.

11. Lin, P. C. and Pearn, W. L. (2002). Testing process capability for one-sided specification limit with application to the voltage level translator. Microelectronics Reliability, 42, 1975-1983.

12. Pearn, W. L. and Chen, K. S. (2002). One-sided capability indices $C_{P U}$ and $C_{P L}$ : decision making with sample information. International Journal of Quality and Reliability Management, 19, 221-245.

13. Pearn, W. L. and Shu, M. H. (2003). An algorithm for calculating the lower confidence bounds of $C_{P U}$ and $C_{P L}$ with application to low-drop-out linear regulators. Microelectronics Reliability, 43, 495-502.

14. Vännman, K. (1998). Families of capability indices for one-sided specification limits. Statistics, 31, 43-66.

15. Vännman, K. (2001). A graphical method to control process capability. Frontiers in Statistical Quality Control, No 6, Editors: Lenz, H.-J. \& Wilrich, P.-TH. Physica-Verlag, Heidelberg, 290-311.

16. Vännman, K. (2006). Safety regions in process capability plots. Quality Technology \& Quantitative Management, 3, 227-246.

17. Vännman, K. and Albing, M. (2007). Process capability indices for one-sided specification intervals and skewed distributions. To appear in Quality and Reliability Engineering International.

18. Vännman, K and Hubele, N. F. (2003). Distributional properties of estimated capability indices based on subsamples. Quality and Reliability Engineering International, 19, 111-128. 


\section{Appendix}

\section{Appendix $A$}

Here the studied families of capability indices in (6) and (7) are expressed as function of $\mu_{t}$ and $\sigma_{t}$. Furthermore, $\sigma_{t}$ is expressed as a function of $\mu_{t}$ for all possible values of the given index value $k$. See Table 3 and Table 4 . We assume that $\mu<U S L$, which is equivalent to $\mu_{t}<1$. Under this assumption the indices $C_{p a u}(0, v)$, for $v \geq 0$, and $C_{p v u}(0, v)$, for $v>0$, will always be positive, i.e. have $k>0$.

By letting $\sigma_{t}$ tend to 0 we can easily show that $C_{p a u}(1, v)$ and $C_{p v u}(1, v)$, for $v>0$, are always larger than $-1 /(3 \sqrt{v})$. Hence $k>-1 /(3 \sqrt{v})$.

Table 3. $C_{p a u}(u, v)$ for $u=0,1$ and $v \geq 0$ for all possible values of $k$.

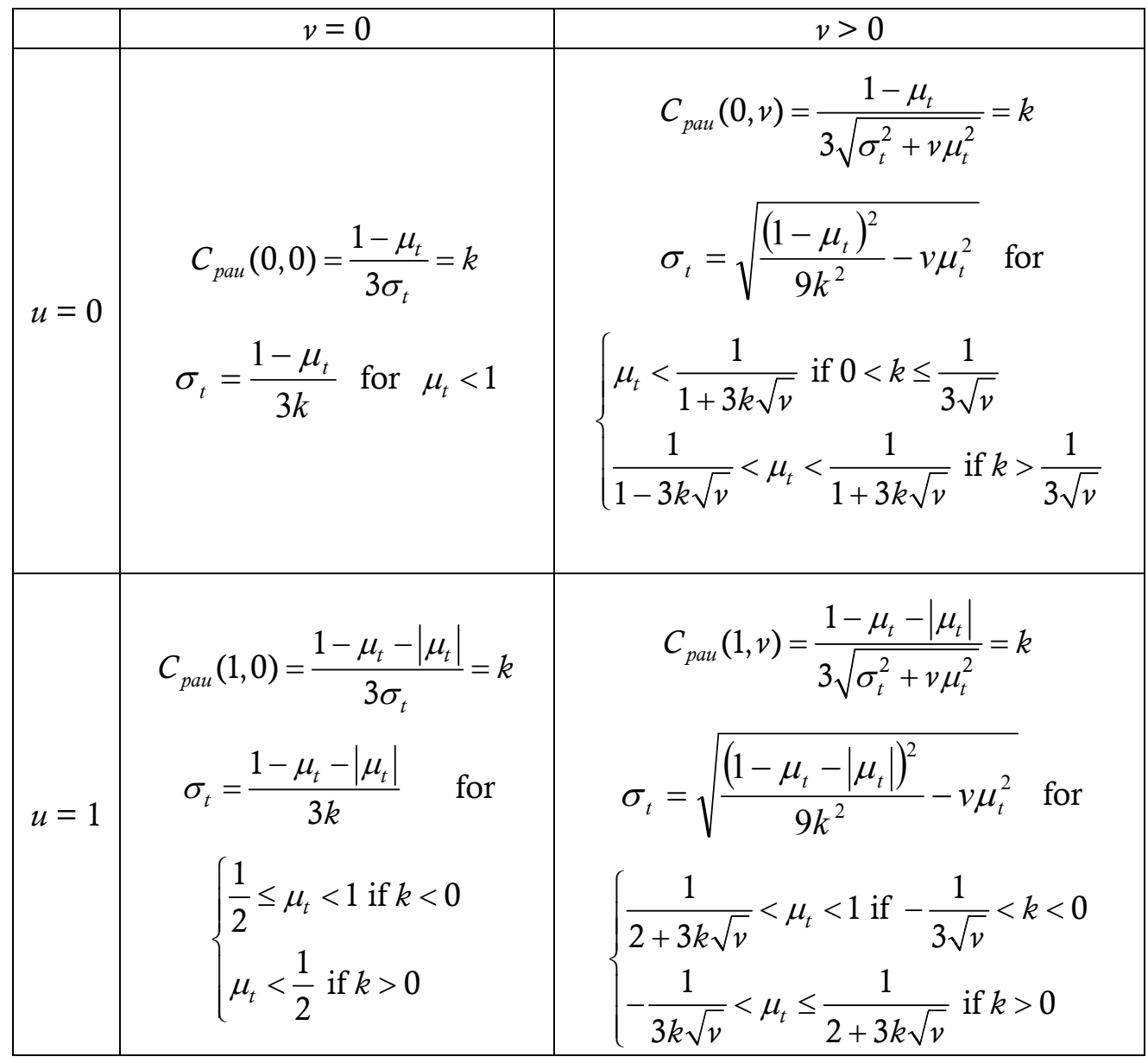


Table 4. $C_{p v u}(u, v)$ for $u=0,1$ and $v>0$ for all possible values of $k$.

\begin{tabular}{|c|c|c|}
\hline & $v=0$ & $v>0$ \\
\hline$u=0$ & $\begin{array}{l}C_{p v u}(u, v) \text { is not defined for } \\
(u, v)=(0,0)\end{array}$ & $\begin{array}{c}C_{p v u}(0, v)=\frac{1}{3 \sqrt{\sigma_{t}^{2}+v \mu_{t}^{2}}}=k \\
\sigma_{t}=\sqrt{\frac{1}{9 k^{2}}-v \mu_{t}^{2}} \text { for } \\
\left\{\begin{array}{l}-\frac{1}{3 k \sqrt{v}}<\mu_{t}<1 \text { if } 0<k \leq \frac{1}{3 \sqrt{v}} \\
\left|\mu_{t}\right|<\frac{1}{3 k \sqrt{v}} \text { if } k>\frac{1}{3 \sqrt{v}}\end{array}\right.\end{array}$ \\
\hline$u=1$ & $\begin{array}{c}C_{p v u}(1,0)=\frac{1-\left|\mu_{t}\right|}{3 \sigma_{t}}=k \\
\sigma_{t}=\frac{1-\left|\mu_{t}\right|}{3 k} \text { for } \\
\left\{\begin{array}{l}\mu_{t}<-1 \text { if } k<0 \\
-1<\mu_{t}<1 \text { if } k>0\end{array}\right.\end{array}$ & $\begin{array}{c}C_{p v u}(1, v)=\frac{1-\left|\mu_{t}\right|}{3 \sqrt{\sigma_{t}^{2}+v \mu_{t}^{2}}}=k \\
\sigma_{t}=\sqrt{\frac{\left(1-\left|\mu_{t}\right|\right)^{2}}{9 k^{2}}-v \mu_{t}^{2}} \text { for } \\
\mu_{t}<-\frac{1}{1+3 k \sqrt{v}} \text { if }-\frac{1}{3 \sqrt{v}}<k<0 \\
\left|\mu_{t}\right|<\frac{1}{1+3 k \sqrt{v}} \text { if } k>0\end{array}$ \\
\hline
\end{tabular}

\section{Appendix B}

We here give the MATLAB code for obtaining the critical value $c_{\alpha}$, for given values of $u, v, n, \alpha$, and $k_{0}$. First the value of $\mu_{t}$, along the contour curve $C_{p u}(u, v)=k_{0}$, that maximize the probability $P\left(\hat{C}_{p u}(u, v)>c_{\alpha} \mid C_{p u}(u, v)=k_{0}\right)$ is obtained, using (9) and (22) or (10) and (25), respectively. Then, for that $\mu_{t}$ value, $c_{\alpha}$ is determined so that $P\left(\hat{C}_{p u}(u, v)>c_{\alpha} \mid C_{p u}(u, v)=k_{0}\right)=\alpha$. As mentioned in Section 2, $C_{p u}(u, v)$ denotes either of the two classes of indices $C_{p a u}(u, v)$ in (6) and $C_{p v u}(u, v)$ in (7).

The user also has to give input values for an interval $\left[x_{0}, x_{1}\right]$, in which the critical value will be located, and the iteration indices $N_{1}-N_{4}$. It is reasonable to choose $x_{0}=k_{0}$ as a first approximation of $x_{0}$. For $x_{1}$ we recommend a fairly large value as a first approximation of $x_{1}$. In order to receive a narrower interval one can run the routine for a small number of the iteration indices $N_{1}-N_{4}$ and thereby find an approximate value of $c_{\alpha}$ and construct a new interval around that value.

For a given value of $x$ the probability $P\left(\hat{C}_{\text {pua }}(u, v)>x\right)$ will be calculated for $N_{4}$ points along the contour curve $C_{p u}(u, v)=k_{0}$. The value of $\mu_{t}$ corresponding to the point along the contour curve $C_{p u}(u, v)=k_{0}$ that gives the largest probability will be identified. Then the probability will be calculated for a new interval around the $\mu_{t}$ value that gave maximum. This will be repeated $N_{3}$ times. This procedure will be executed for the $N_{2}$ points in the interval $\left[x_{0}, x_{1}\right]$ and the $x$ value that gives the largest probability $P\left(\hat{C}_{p u a}(u, v)>x\right)$ will be identified. Then a new interval $\left[x_{0}, x_{1}\right]$ is constructed around that $x$-value, i.e. the 
one that gave the largest probability, and the probability $P\left(\hat{C}_{p u a}(u, v)>x\right)$ is calculated for this new interval. This will be repeated $N_{l}$ times and the critical value will be defined as the midpoint of the final interval.

The MATLAB code here is given for $C_{p a u}(u, v)$, where $u \geq 0$ and $v>0$. In order to receive the corresponding code for $C_{p a u}(u, 0), C_{p v u}(1,0)$ and $C_{p v u}(u, v)$, where $v>0$, respectively, we replace the restrictions of $\mu_{t}$ in (a) by the restrictions in (9) and (10), respectively. Furthermore we replace $\sigma_{t}$ in (b) by (9) and (10) and the probability in (c)-(d) by formula (22) and (24), and (25) respectively.

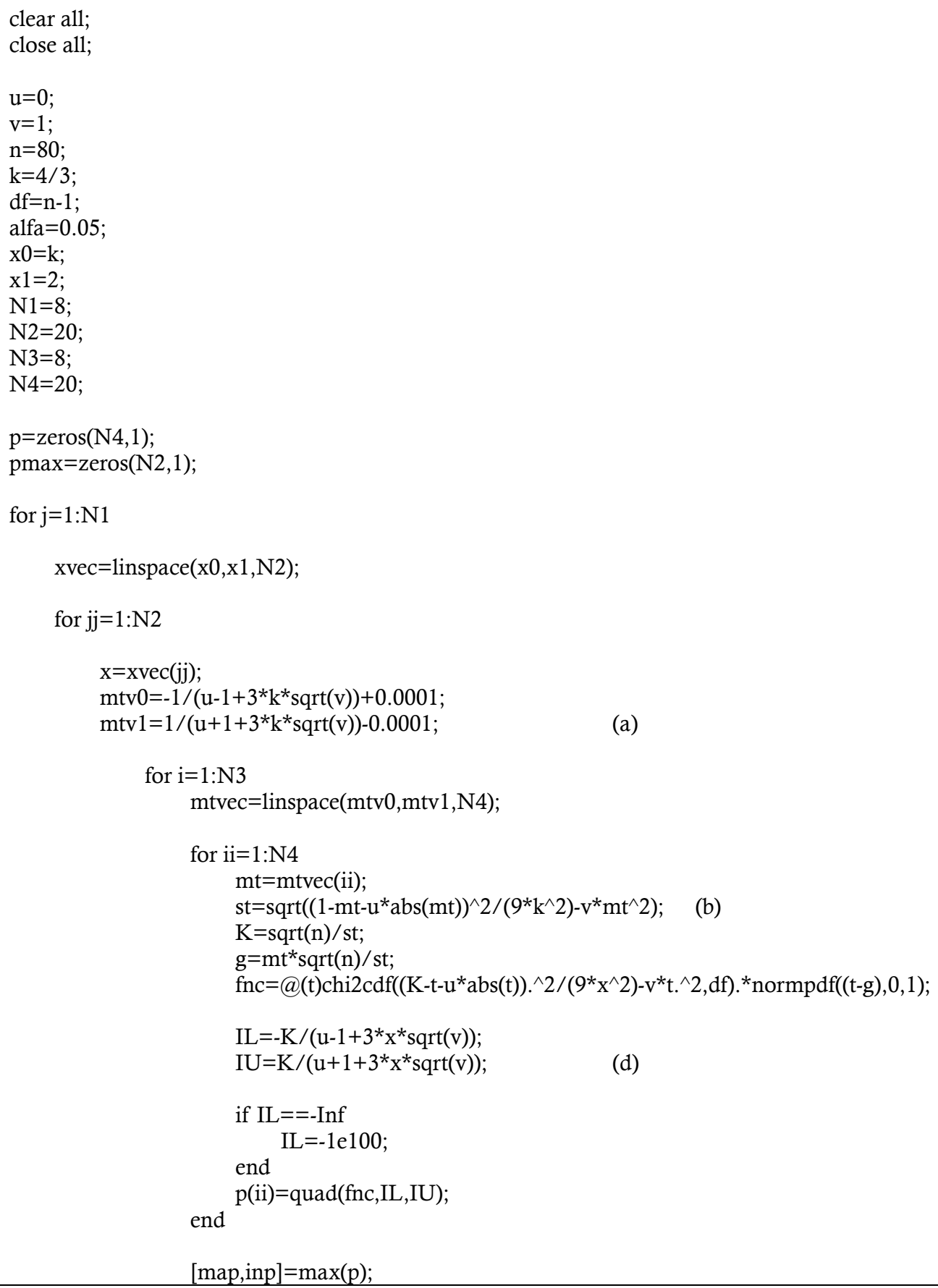




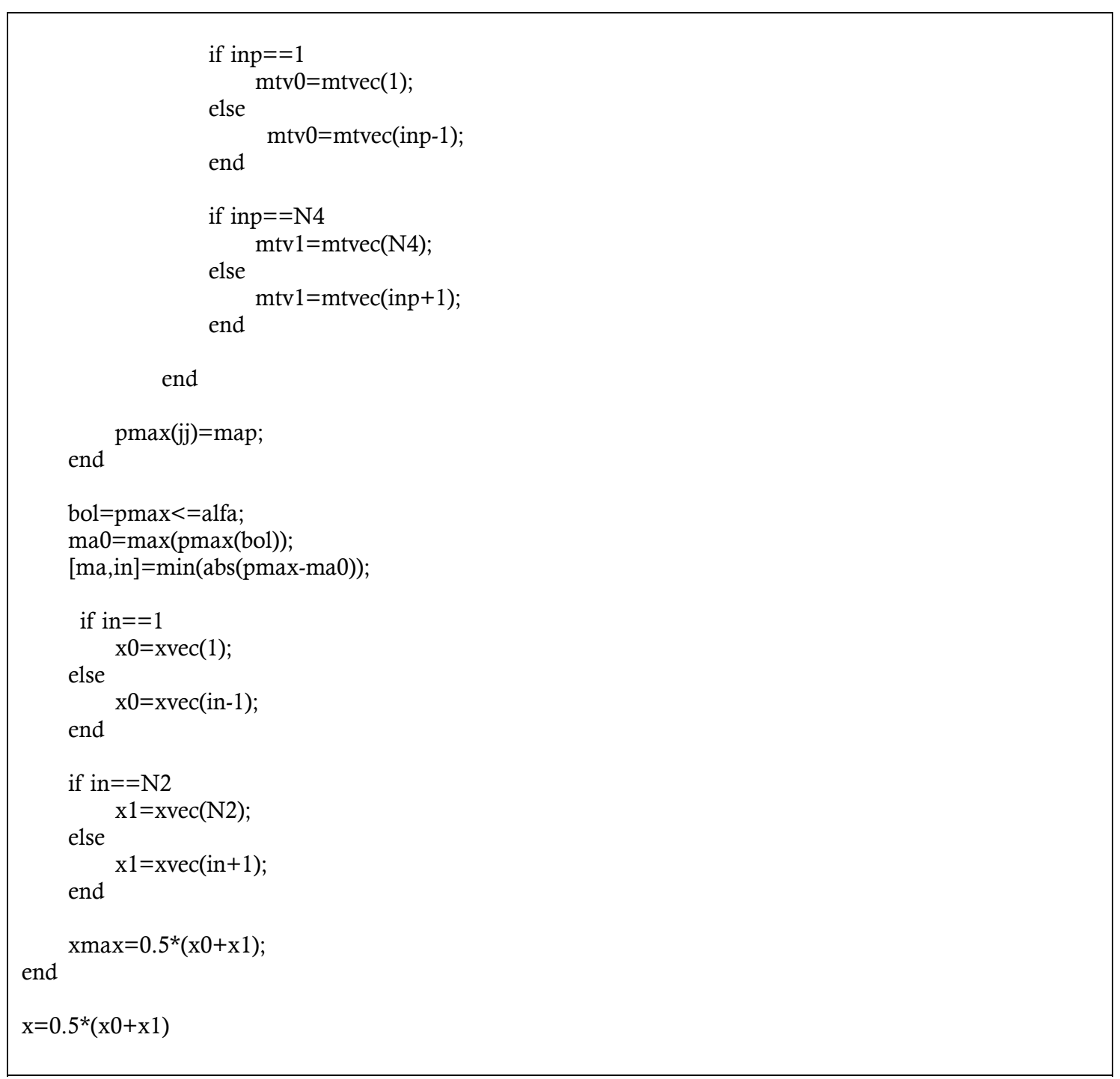

\section{Authors' Biographies}

Kerstin Vännman is a Professor in Statistics with special emphasis on Industrial Statistics at the Department of Mathematics, Luleå University of Technology, Luleå, Sweden. Her main research interest is currently in the field of process capability analysis and statistical process control, but she has also an interest in statistical education, with publications in both fields. She is a member of ISI and ASA.

Malin Albing is $\mathrm{Ph} \mathrm{Lic} \mathrm{in} \mathrm{Statistics} \mathrm{with} \mathrm{special} \mathrm{emphasis} \mathrm{on} \mathrm{Industrial} \mathrm{Statistics} \mathrm{at} \mathrm{the}$ Department of Mathematics, Luleå University of Technology, Luleå, Sweden. Her main research interest is currently in the field of process capability analysis. 